\title{
Analytical profiling and structure elucidation of flavanoids compounds
}

\author{
Available online at www.ijistweb.com \\ RESEARCH ARTICLE \\ Rajni Singh Khureja*, Dinesh Kumar \\ Department of Chemistry, University Teaching Department, Sri Satya Sai University of \\ Technology \& Medical Sciences, Pachama, Sehore-466001-MP, India. \\ *Corresponding Author's E-mail: om11agra85@gmail.com
}

DOI: $10.22270 /$ ijist.v4i1.35

\begin{abstract}
Chromatography is a powerful analytical method suitable for the separation and quantitative determination of a considerable number of compounds, even from complicated matrix. Thin Layer Chromatography (TLC) has some advantages such as rapidity, sensitivity, easiness, cheapness and this method does not require complex instrumental equipment. In present study, Quercetin $3-\mathrm{O}-\alpha$ - d- glucuro pyranoside (miquelianin; QG), quercetin 3-O- $\alpha$-d glucopyranoside (isoquercitrin), quercetin 3-O- $\alpha$-d-galactopyranoside (hyperoside) and rutin in ethyl acetate fractions from aerial parts of selected Potentilla species on a HPTLC plates using a mixture consisting of ethyl acetate/methyl ethyl ketone/diisopropyl ether/formic acid (3:10:4:1, v/v/v/v). Rutinosides and quercetin were also eluted using methanol-water-acetic acid (50/44/6, v/v/v) and benzene: pyridine: formic acid (36:9:5). The spot visualization was evaluated under UV light at $254 \mathrm{~nm}$ and Ferric chloride reagent.
\end{abstract}

Keywords: Flavanoid, HPTLC, HPLC, Qurecin, Elucidation.

\section{Introduction}

Natural products (secondary metabolites) have been the most successful source of potential drug leads. However, their recent implementation in drug discovery and development efforts have somewhat demonstrated a decline in interest. Nevertheless, natural products continue to provide unique structural diversity in comparison to standard combinatorial chemistry, which presents opportunities for discovering mainly novel low molecular weight lead compounds. Since less than $10 \%$ of the world's biodiversity has been evaluated for potential biological activity, many more useful natural lead compounds await discovery with the challenge being how to access this natural chemical diversity (1-3).

The earliest records of natural products were depicted on clay tablets in cuneiform from Mesopotamia (2600 B.C.) which documented oils from Cupressus sempervirens (Cypress) and Commiphora species (myrrh) which are still used today to treat coughs, colds and inflammation. The Ebers Papyrus (2900 B.C.) is an Egyptian pharmaceutical record, which documents over 700 plant-based drugs ranging from gargles, pills, infusions, to ointments. The Chinese Materia Medica (1100 B.C.) (Wu Shi Er Bing Fang, contains 52 prescriptions), Shennong Herbal ( 100 B.C., 365 drugs) and the Tang Herbal (659 A.D., 850 drugs) are documented records of the uses of natural products $^{4-5}$. The Greek physician, Dioscorides, (100 A.D.), recorded the collection, storage and the uses of medicinal herbs, whilst the Greek philosopher and natural scientist, Theophrastus ( 300 B.C.) dealt with medicinal herbs. During the Dark and Middle Ages the monasteries in England, Ireland, France and Germany preserved this Western knowledge whilst the Arabs preserved the Greco-Roman knowledge and expanded the uses of their own resources, together with Chinese and Indian herbs unfamiliar to the Greco-Roman world. It was the Arabs who were the first to privately own pharmacies ( $8^{\text {th }}$ century) with Avicenna, a Persian pharmacist, physician, philosopher and poet, contributing much to the sciences of pharmacy and medicine through works such as the Canon Medicinae (6-7).

The study of natural products has had a number of rewards. It has led to the discovery 
of a variety of useful drugs for the treatment of diverse ailments and contributed to the development of separation science and technology, spectroscopic methods of structure elucidation and synthetic methodologies that now make up the basics of analytical organic chemistry (8-10).

Currently many modern medicines are products of nature or derived from natural products, and nature is a promising source of treatments for new and emerging medical conditions. The search for treatments for diseases or infection from nature preceded the discovery of new technologies like ultra-high throughput screening (uHTS), combinatorial chemistry, and genomic technology. Drug discovery from natural products has however been impacted significantly by the evolution of these new technologies, and there is an emerging perception that the role of natural products is starting to diminish. In spite of this, plants are still capable of yielding new bioactive compounds, and microbial organisms continue to yield novel structures and novel bioactivities. Since only $10 \%$ of the world's biodiversity has been studied for potential curative entities, the remainder is waiting to be welcomed to the world of drug discovery (11).

The term-flavonoid is generally used to describe a broad collection of natural products that include a $\mathrm{C}_{6}-\mathrm{C}_{3}-\mathrm{C}_{6}$ carbon framework, or more specifically phenylbenzopyran functionality (12). Flavonoids are a class of plant phenolics that comprise an astonishingly diverse group of more than 4500 compounds and derived from the condensation of a cinnamic acid with three malonyl-CoA groups (Figure.2.1). All flavonoids arise from the initial reaction, which is catalyzed by the chalone synthase enzyme (13).

So, in present study, we investigate structure elucidate of isolated compounds selectively for Flavonoids structure.

\section{Materials and methods}

The flavonoid content in plants is strongly influenced by extrinsic factors such as variations in plant type and growth, season, climate, degree of ripeness, food preparation and processing. Flavonoids have a wide range of biological activities, such as inhibition of cell- proliferation, inducing-apoptosis, enzyme inhibiting activity, antibacterial, and antioxidant effects. Moreover, some findings indicate that flavonoids possess various clinical properties, such as anti-atherosclerotic, anti-inflammatory, anti-tumor (), antithrombogenic, anti-osteoporotic, and antiviral effects. Flavonoids decrease the risk of coronary heart disease by three major actions: improving coronary vasodilatation, decreasing the ability of platelets in the blood to clot, and preventing low-density lipoproteins (LDLs) from oxidizing. These poly-phenols interact with $\mathrm{ABC}$ drug transporters involved in drug resistance and drug absorption, distribution and excretion.

\section{Extraction of Flavonoids}

Most of flavonoids are extracted readily from source material by alcohol or alcohol/ water mixtures. Prior knowledge of the nature of the flavonoids is helpful in determining the most efficient extraction techniques. The extraction method allows to concentrate and to clean up the extracted sample before chromatographic injection and during the extraction of plant material, it is important to minimize interfering compounds that may co-extract with the target compounds, and to avoid contamination of the extract, as well as to prevent decomposition of important metabolites or solvent impurities.

Method of Soxhlet extraction is not always acceptable for industrial applications due to long extraction time, and large consumption of hazardous solvents. This extraction method is also not suitable for the extraction of thermosensitive compounds due to the probability of thermal decomposition of target compounds as extraction usually occurs at the boiling point of used solvent for a long time.

\section{Quantification of Flavonoids}

The total flavonoid content in extractive solvents represents an important parameter in evaluating the extractive process and is frequently based on the complexation with aluminium chloride or based on the formation of terbium $\left(\mathrm{Tb}^{3+}\right.$ )-flavonoids (quercetin as a reference standard) complex at $\mathrm{pH} 7.0$, which has fluorescence intensely with maximum emission at $545 \mathrm{~nm}$ when excited at $310 \mathrm{~nm}$. 
The methods of determination of chemical composition of plant extracts include preliminary chromatographic separation, thin layer chromatography (TLC), High performance chromatography (HPLC) and preparative thin layer chromatography, and hyphenated techniques such as Nuclear Magnetic Resonance spectrophotometry (NMR). These first three methods were used for rapid initial screening of crude plant extract, and provide initial information on the content and the nature of constitutions in the matrix. Chromatographic methods (thin layer chromatography and high performance liquid chromatography) were used for identification, quantification, and characterization of individual flavonoid or phenolic acid.

\section{Results and discussion}

Quercetin O-glycosides are quercetin derivatives with at least one $\mathrm{O}$-glycosidic bond which are widely distributed in the plant kingdom. A quercetin glycoside is formed by attaching a glycosyl group (a sugar such as glucose, rhamnose, or rutinose) as a replacement for one of the $\mathrm{OH}$ groups (commonly at position 3 ). The attached glycosyl group can change the solubility, absorption, and in vivo effects of quercetin glycosides. Quercetin 7-O- rhamnoside reduces porcine epidemic diarrhea virus replication via independent pathway of viral induced reactive oxygen specie. Isoquercetin (quercetin 3-O-glucoside) had a regulative role in blood glucose level and lipids, which improved the function of pancreatic islets, and may be useful in the treatment of type 2 diabetes mellitus. The chelating site of quercetin involves the deprotonated $\mathrm{C}_{5}-\mathrm{OH}$ and the $\mathrm{C}_{4}=\mathrm{O}$ groups at ring $\mathrm{C}$ of quercetin. Leishmanicidal flavonol quercetin is a mixed inhibitor whereas quercitrin and isoquercitrin are uncompetitive inhibitors of Leishmania (Leishmania) amazonensis arginase.
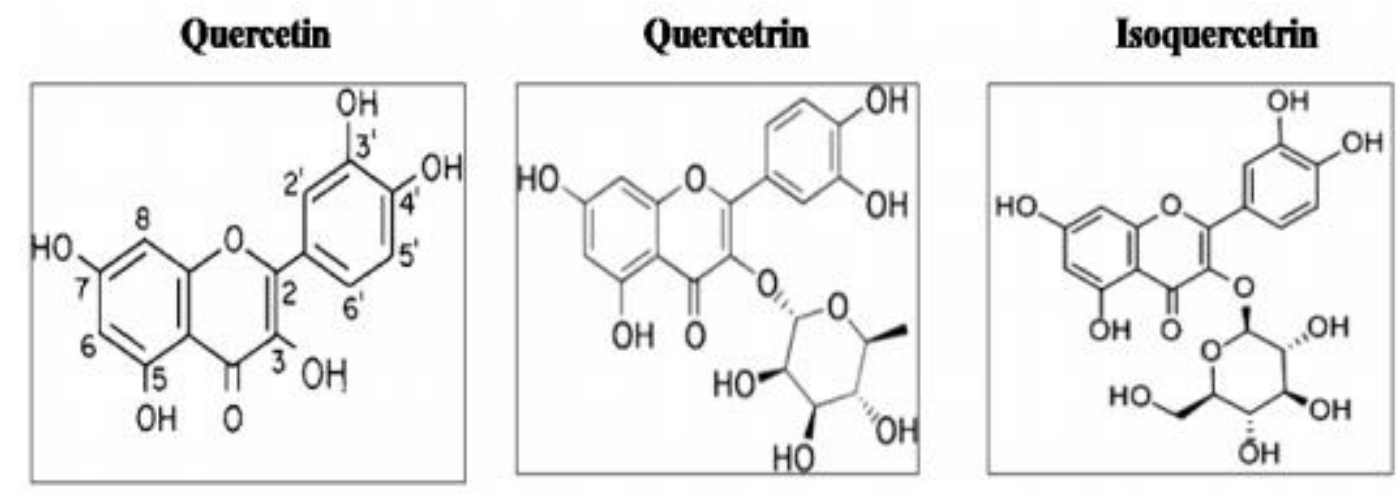

Rutin

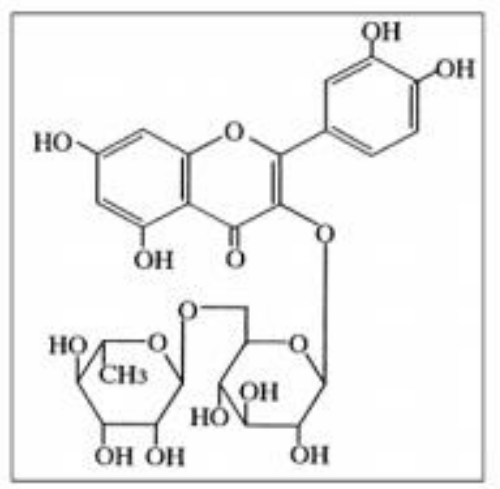

Initially, Quercetin methyl ether derivatives displayed cytotoxic properties, which induced $\mathrm{G}_{2}-\mathrm{M}$ phase cell cycle arrest and apoptosis on human myeloid leukemia cells and nowadays, these are used for treatment of inflammatory diseases related with NO overproduction due 
to inducible nitric oxide synthase. Quercetin3-gallate showed comparable antiviral activity against influenza virus and no cytotoxic effects on Caucasian hepatocyte or lung carcinoma at concentrations as high as $200 \mu \mathrm{M}$. Nowadays, Quercetin derivatives act as novel antibacterial agents by showing dual inhibition on DNA (14) gyrase and topoisomerase.

Structural modification of quercetin by methylation at the $5^{\text {th }}$ or $7^{\text {th }}$ position is the most optimal to retain most of the antioxidant capacity of quercetin and it protect brain from disorders associated with oxidative stress. They interact with lipid bilayers, and play a role of- guarding molecules, preventing penetration of acyl chain regions by reactive oxygen species. Thapa et al. (2012) investigated the synthesis and antiviral activities of various quercetin derivatives with substitution of $\mathrm{C}_{3}$ and $\mathrm{C}_{5}$ hydroxyl functions with various phenolic ester, alkoxy, and amino alkoxy moieties (13).

\section{Health Effects of Quercetin}

For decades, quercetin has been widely investigated, mainly because of its putative health-promoting effects, for example as a potential protection against coronary heart disease and hypertension. These health claims are merely supported by in vitro evidence related to the cytoprotective ability of quercetin to modulate the activity of numerous enzymes involved in energy metabolism, signal transduction, cell growth and antioxidant activity. Quercetin is an emerging prospective anticancer drug candidate and its pro-drug $\mathrm{QC}_{12}$ has entered phase-I clinical studies. Quercetin showed neuronal cell protective effect against glutamate-induced neurotoxicity, and recovered the mitotic index and chromosomal instability after treatment with hydrogen peroxide.

\section{Bioavailability of Quercetin}

Bioavailability studies performed with quercetin have recently been reviewed and showed that the rate of elimination of quercetin metabolites is relatively slow, with half-lives ranging from 11 to 28 hours. This could favour accumulation in plasma with repeated intakes. Quercetin, and possibly other related flavonoids, given orally are rapidly metabolized in the intestine and liver into glucuronidated derivatives which act as carriers of quercetin and deliver the free aglycone in situ by deconjugation. Moreover, the intravenous administration of quercetin-3O-glucuronide resulted in a slow onset and sustained blood pressure lowering effect.

\section{In Vitro Culture Techniques}

Plant cell/tissue culture, also referred to as in vitro, axenic, or sterile culture, is an important tool in commercial application for production of whole plants. The capacity for plant cell, callus, and organ cultures to produce and accumulate many of the same valuable chemical compounds as the parent plant in nature has been recognized almost since the inception of in vitro technology. Callus cultures, containing more or less homogenous clumps of dedifferentiated cells, are used for secondary metabolite production.

The production of compact callus at cut edge of explant may be due to the wound during the process of cutting which resolved in a synchronous cell division. Changes in hormone composition provoke marked perturbations in growth and biosynthetic characteristics of the cultures. The size of callus clumps may determine the chemical gradients that influence chemical synthesis. However, many factors such as genotype, composition of the nutrient medium, and physical growth factors such as light, temperature, humidity, and endogenous supply of growth regulators are important for callus induction.

Under in vitro conditions, explants have been removed from their original tissue environment and transferred to synthetic media containing non-physiological concentrations of growth regulators and organic and inorganic constituents, resulting in exposure to significant stresses. Plant growth regulator can be defined as either natural or synthetic compounds that modify the plant growth and development pattern exerting profound influence on many physiological processes and involved in the regulation of these developmental switches under in vitro conditions. Auxins, cytokinins, gibberellins, abscisic acid and ethylene are commonly 
recognized as naturally occurring plant hormones.

Morphogenetic response of leaf discs- explants was greatly influenced by the type of growth regulator used in the medium. Different plant hormones like 2, 4-D, kinetin, IAA, BAP influence callus induction from explants. For continuity of callus induction, 2, 4-D alone or in combination with $K_{n}$ are essential and auxin reduction may lead to organogenesis and embryo formation. Changes in hormone composition provoke marked perturbations in growth and biosynthetic characteristics of the cultures. As callus growing in a nutrient-rich culture medium undoubtedly exposed to more carbon influx than the field- grown plant parts, it may influence the metabolic flux for the biosynthesis of elevated levels of phenols. The age of the cells in the inoculum also had influence on metabolite production and determines the way the culture grows.

Established callus cultures from the different parts of Maackia amurensis and analyzed for isoflavonoids. The isoflavones daidzein, retuzin, genistein, formononetin, and the pterocarpans maakiain were found to be produced by these cultures. Shashikala et al. (2009), studied callus induction from leaf explants of Centella asiatica and leaf callus showed high accumulation of asiaticoside compared to parent plant. Quercetin production also observed in callus cultures, biosynthesis of apigenin, kaempferol, quercetin were identified from Indigofera cordifolia callus cultures.

\section{Acknowledgement}

The authors are thankful to IJIST Journal for publishing their article

\section{Conflicts of Interest}

The author declares that there are no conflicts of interest.

\section{References}

1. Wolfender J-L., Ndjoko K., Hostettmann K. Liquid chromatography with ultraviolet absorbance-mass spectrometric detection and with nuclear magnetic resonance spectroscopy: a powerful combination for the on-line structural investigation of plant metabolites. Journal of chromatography A. $2003 ; 1000: 437-455$.

2. Reynolds W. F., Enrìquez R. G. Choosing the best pulse sequences, acquisition parameters, postaquisition processing strategies, and probes for natural product structure elucidation by NMR spectroscopy. Journal of natural products. 2002; 65:221-244.

3. Marston A., Hostettmann K. Natural product analysis over the last decades. Planta medica. 2009; 75:672-682.

4. Kind T., Fiehn O. Advances in structure elucidation of small molecules using mass spectrometry. Bioanalytical reviews. 2010; 2:23-60.

5. Stroh J. G., Petucci C. J., Brecker S. J., Huang N., Lau J. M. Automated sub-ppm mass accuracy on an ESI-TOF for use with drug discovery compound libraries. Journal of American society for mass spectrometry. 2007; 18:1612-16.

6. Hu Q., Noll R. J., Li H., Makarov A., Hardman M., Cooks G. The Orbitrap: a new mass spectrometer. Journal of mass spectrometry. 2005; 40:430-43.

7. Lu W., Bennet B. D., Rabinowitz J. D. Analytical strategies for LC-MS-based targeted metabolomics. Journal of chromatography B. 2008; 871:236-42.

8. Glish G. L., Vachet R. W. The basics of mass spectrometry in the twenty-first century. Nature reviews drug discovery. 2003; 2:140-150.

9. Bhowmick M., Bhowmick P., Sengodan T., Thangavel, S. Development and validation of bioanalytical RP HPLC method for the estimation of metoprolol tartrate in rabbit plasma after transdermal and oral administration: application in pharmacokinetic studies. Journal of Drug Delivery and Therapeutics. 2015; 5(4):4353.

10. Bhatt P, Vhora I, Patil S, Amrutiya J, Bhattacharya C, Misra A, et al. Role of antibodies in diagnosis and treatment of ovarian cancer: Basic approach and clinical status. Journal of controlled release : official journal of the Controlled Release Society. 2016;226:148-67.

11. Bilia A. R., Bergonzi M. C., Mazzi G., Vincieri F. F. Analysis of plant complex 
matrices by use of nuclear magnetic resonance spectroscopy: St. John's wirt extract. Journal of agricultural and food chemistry. 2001; 49:2115-24.

12. Cos A., Vlietinck A. J., Berghe D. V., Maes L. Anti-infective potential of natural products: How to develop a stronger in vitro proof-of-concept. Journal of ethnopharmacology. 2006; 106:290-302.

13. Sasidharan S., Chen Y., Saravanan D., Sundram K. M., Latha L. Y. Extraction, isolation and characterization of bioactive compounds from plants'extracts. African journal of traditional, complementary and alternative medicine. $2011 ; 8(1): 1-1$
14. Bhatt P, Lalani R, Mashru R, Misra A. Abstract 2065: Anti-FSHR antibody Fab' fragment conjugated immunoliposomes loaded with cyclodextrin-paclitaxel complex for improved <em>in vitro</em> efficacy on ovarian cancer cells. Cancer Research. 2016;76(14 Supplement):2065-.

15. Bhatt P, Khatri N, Kumar M, Baradia D, Misra A. Microbeads mediated oral plasmid DNA delivery using polymethacrylate vectors: an effectual groundwork for colorectal cancer. Drug delivery. 2015;22(6):849-61. 